\title{
Interaction of Large-Scale Equatorial Waves and Dispersion of Kelvin Waves through Topographic Resonances
}

\author{
ANDREW J. MAJDA \\ Courant Institute of Mathematical Sciences, New York University, New York, New York \\ RODOLFO R. ROSALES \\ Department of Mathematics, Massachusetts Institute of Technology, Cambridge, Massachusetts \\ ESTEBAN G. TABAK \\ Courant Institute of Mathematical Sciences, New York University, New York, New York \\ CRISTINA V. TURNER \\ F.A.M.A.F., University of Cordoba, Cordoba, Argentina
}

(Manuscript received 4 September 1997, in final form 17 December 1998)

\begin{abstract}
A new theoretical mechanism is developed in which large-scale equatorial Kelvin waves can modify their speed through dispersion and interaction with other large-scale equatorial waves, such as Yanai or Rossby modes, through topographic resonance. This resonance mechanism can prevent the breaking of a propagating nonlinear Kelvin wave, slow down its speed, and concentrate most of its energy in large-scale zonal wavenumbers while simultaneously generating large-scale Yanai or Rossby modes with specific zonal wavelengths. Simplified reduced dynamic equations for this resonant interaction are developed here via suitable asymptotic expansions of the equatorial shallow water equations with topography. Explicit exact solutions for the reduced equations and numerical experiments are utilized to display explicitly the features of large-scale dispersion and topographic resonance for equatorial Kelvin waves mentioned earlier. Two examples of this theory, corresponding to the barotropic and first baroclinic modes of the equatorial troposphere, are emphasized.
\end{abstract}

\section{Introduction}

Large-scale eastward-propagating waves are a distinctive feature of the intraseasonal Madden-Julian oscillation (Madden and Julian 1972, 1994) of the equatorial troposphere. Current theories attempt to explain these modes as essentially linear or nonlinear Kelvin waves of equatorial shallow water models representing the first baroclinic mode with suitable convective parameterization (Emanuel 1987; Neelin et al. 1987; Wang and Xue 1992; Neelin and Yu 1994; Yano et al. 1995) through either wave-Cisk or evaporation-wind feedback mechanisms, which slow down the Kelvin waves. There are also observations of large-scale westward-propagating waves embedded within eastward-propagating

Corresponding author address: Prof. Andrew J. Majda, Courant Institute of Mathematical Sciences, New York University, 251 Mercer St., New York, NY 10012.

E-mail: krouhaus@cims.nyu.edu super cloud clusters in the western Pacific and a corresponding linear theory (Goswami and Goswami 1991) for equatorial Yanai or mixed Rossby gravity waves, which provides a possible mechanism for this phenomenon. Large-scale Kelvin and Yanai waves in the troposphere also have a prominent role in the forcing of low-frequency oscillations of the equatorial stratosphere such as the quasi-biennial oscillation (Andrews et al. 1987; Takahashi and Boville 1992).

Here we present a new theoretical mechanism in which large-scale equatorial Kelvin waves can modify their speed through large-scale dispersion and interaction with other large-scale equatorial waves, such as Yanai or Rossby modes, through topographic resonance. This resonance mechanism can also generate simultaneously large-scale Yanai or Rossby modes with specific wavelengths and prevent the breaking of the nonlinear Kelvin wave while concentrating most of its energy in large-scale zonal modes. To clearly elucidate the structure of this new mechanism in the simplest physical context, here the equatorial shallow water equations 
with topography with an equivalent height generated by either the barotropic or first baroclinic mode of the equatorial troposphere are utilized. For dynamics in the equatorial troposphere, this reduction from the full vertical structure to a shallow water model is often done by Galerkin projection onto a single vertical mode of the full primitive equations in pressure coordinates (see, e.g., Neelin and Zeng 1997). Recent EOF analysis of observational data in the western Pacific (Milliff and Madden 1996) provides evidence that the dominant two modes in eastward-propagating equatorial waves are the first baroclinic and barotropic modes, respectively. In this paper the topography is regarded as crudely modeling large-scale land-sea contrasts, but the authors have no doubt that the role of topography elucidated here can be replaced in similar models by other large-scale inhomogeneous mechanisms, such as diabatic heating or evaporation wind feedback with convective parameterization, which accounts for different bulk fluxes for land and sea (Neelin and Zeng 1997). We plan to develop these more elaborate applications in the near future. The reason why the authors believe that these other mechanisms will play similar roles as the topography is that entirely similar behaviors appear in the context of gas dynamics (Majda et al. 1988; Celentano 1995; Vaynblat 1996; Shefter 1997), where the coupling of the waves is through variations in the gas entropy-not an external entity such as the topography. The behaviors presented in this paper, in fact, seem to be common to any situation in which nonlinear nondispersive waves resonate with other waves through variations in the media they propagate in (entropy, humidity, vorticity) or variable coefficients (topography). A brief summary and discussion of the content of the remainder of this paper is presented next.

After preliminary discussion of the equatorial shallow water equations in section $2 \mathrm{a}$, the basic nonlinear resonance mechanism of large-scale equatorial waves through topography is described in section $2 \mathrm{~b}$. Through an asymptotic expansion procedure described in detail in section 5, this topographic resonance mechanism yields suitable simplified reduced equations for the amplitude of interacting large-scale equatorial waves. In section $2 \mathrm{c}$, these equations are presented with an equivalent height corresponding to the barotropic mode. The simplified equations in this case are given by

$$
\begin{aligned}
K_{\tau}+\left(\frac{1}{2} K^{2}\right)_{\theta} & =\gamma\left[Y(\tau) e^{i \theta}+\bar{Y}(\tau) e^{-i \theta}\right] \text { and } \\
Y_{\tau} & =-\gamma \hat{K}(1, \tau),
\end{aligned}
$$

where $K(\theta, \tau)$ is the amplitude of the Kelvin wave, a periodic function of $\theta$, and $Y(\tau)$ is the amplitude of the Yanai wave with wavenumber two while $\gamma$ represents the amplitude of wavenumber three topography [the variables $\tau$ and $\theta$ are rescaled shifted variables (see section 5)]. Thus, the nonlinear Kelvin wave and a specific large-scale Yanai wave interact through topographic resonance with wavenumber three and these are the only wavelengths with such resonance. In Eq. (1.2) the quantity $\hat{K}(1, \tau)$ is the projection onto the first zonal harmonic of the Kelvin wave amplitude. Without topographic resonance, that is, with $\gamma=0$, Eq. (1.1) reduces to the inviscid Burgers equation describing nonlinear Kelvin waves (Boyd 1980; Ripa 1982); however, completely different large-scale dispersive phenomena occur for $\gamma$ $\neq 0$, which are developed in detail in section 3. In section $2 \mathrm{~d}$, we briefly describe the reduced equations analogous to (1.1) and (1.2), which occur for an equivalent height corresponding to the first baroclinic mode; here several large-scale Yanai and equatorial Rossby waves can interact with the Kelvin wave through equations with a similar structure as in (1.1) and (1.2).

In section 3, some basic properties of solutions of these reduced asymptotic equations are studied including large-scale dispersion and phase modification of the Kelvin waves and generation of large-scale Yanai or Rossby waves. The effect of the mean wind on the resonantly interacting waves is presented in section 4, while the asymptotic derivation of the reduced model is presented in section 5 . The asymptotic procedure utilized in section 5 is based on the method of multiple scales and follows similar asymptotic procedures to those that two of the authors (Majda and Rosales 1984) have applied to resonant waves in compressible flow. For the special case of (1.1) with $\gamma=0$, this procedure yields a simpler asymptotic derivation of the nonlinear propagating Kelvin wave than that via strained coordinates (Boyd 1980), which is also different from Ripa's (1982) elegant Galerkin projection technique for deriving the propagation of nonlinear Kelvin waves. In section $5 \mathrm{~b}$, results of a similar analysis with forcing through diabatic heating are briefly discussed, showing that the identical asymptotic equations are valid in this context. This fact helps to motivate and justify the use in this paper of a wave speed for baroclinic Kelvin modes of roughly $50 \mathrm{~m} \mathrm{~s}^{-1}$. This is the typical wave speed assumed for convective forcing of the troposphere in single-layer models (Gill 1982; Yano et al. 1995; Milliff and Madden 1996).

\section{The reduced model equations}

a. Basic equations, nondimensionalization, and linear waves

The nondimensionalized equations for shallow water in the equatorial waveguide are given by

$$
\begin{aligned}
\eta_{t}+[(1+\eta-h) u]_{x}+[(1+\eta-h) v]_{y} & =0, \\
u_{t}+u u_{x}+v u_{y}+\eta_{x}-y v & =0, \quad \text { and } \\
v_{t}+u v_{x}+v v_{y}+\eta_{y}+y u & =0 .
\end{aligned}
$$

Here the total dimensional depth of the fluid is 


$$
\frac{c^{2}}{g}(1+\eta-h)
$$

where $c$ is the characteristic speed of the linear waves, $g$ is the acceleration of gravity, $\eta$ is the nondimensional perturbation of the free surface, and $h$ is the nondimensional topography. The $x$ and $y$ velocities $u$ and $v$ are nondimensionalized by the characteristic speed $c$, and the spatial variables by the scale

$$
L=\left(\frac{c}{\beta}\right)^{1 / 2}
$$

where $\beta$ is the linear variation of the Coriolis parameter with latitude, given by

$$
\beta=\frac{2 \Omega}{R}
$$

Here $\Omega=(2 \pi) / 24 h s$ is the angular velocity of the earth, with $R=6378 \mathrm{~km}$ the radius. The timescale $T$ is given by

$$
T=\frac{L}{c} .
$$

The solutions to the linearization of the equations above can be divided into two groups: a nondispersive Kelvin wave; and an infinite set of dispersive waves, the Rossby, Yanai, and Poincaré waves (Gill 1982). The Kelvin wave is given by

$$
\begin{aligned}
\eta & =K(x-t) e^{-\left(y^{2} / 2\right)}, \\
u & =K(x-t) e^{-\left(y^{2} / 2\right)}, \quad \text { and } \\
v & =0,
\end{aligned}
$$

where $K$ is an arbitrary function. The dispersive waves have the general form

$$
\begin{aligned}
& \eta=\left[\frac{y}{k-\omega} H_{n}(y)+\frac{\omega}{\omega^{2}-k^{2}} H_{n}^{\prime}(y)\right] e^{i(k x-\omega t)} e^{-\left(y^{2} / 2\right),} \\
& u=\left[\frac{y}{k-\omega} H_{n}(y)+\frac{k}{\omega^{2}-k^{2}} H_{n}^{\prime}(y)\right] e^{i(k x-\omega t)} e^{-\left(y^{2} / 2\right)},
\end{aligned}
$$

and

$$
v=i H_{n}(y) e^{i(k x-\omega t)} e^{-\left(y^{2} / 2\right)},
$$

where $H_{n}(y)$ is the Hermite polynomial of order $n$, and $\omega=W(k)$ satisfies the dispersion relation

$$
-2 n+\frac{(\omega+k)\left(\omega^{2}-k \omega-1\right)}{\omega}=0 .
$$

The solutions with positive $\omega$ to this cubic equation are displayed in Fig. 1. The case with $n=0$ has only two solutions, corresponding to the Yanai (or mixed Rossby gravity) wave; the third solution to the cubic $(\omega=-k)$ is spurious because it does not satisfy the original equations. For $n \geq 1$, the solutions are one Rossby and two

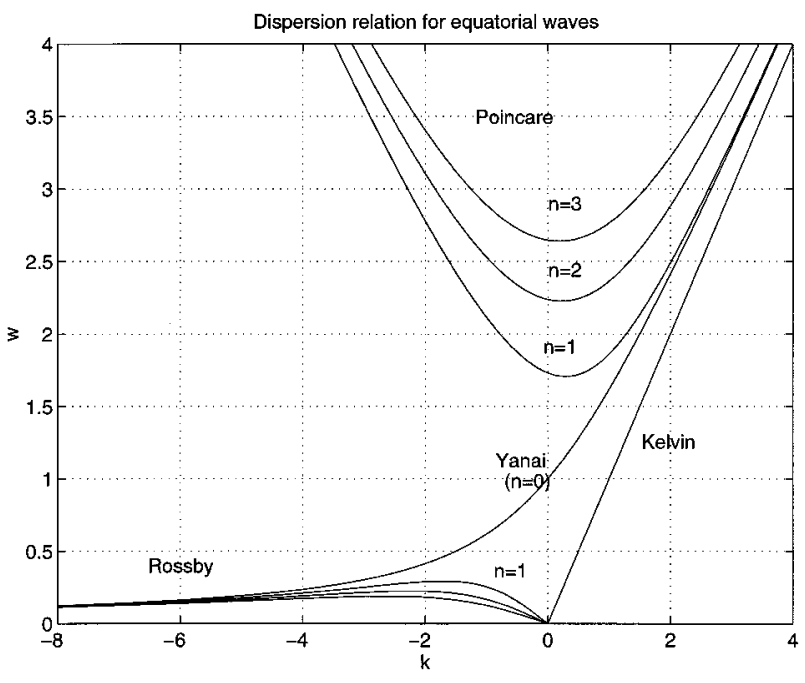

FIG. 1. Dispersion relation for equatorial waves with curves corresponding to the Kelvin, Yanai, and the first few Rossby and Poincaré waves. Since the dispersion relation is skew-symmetric, only the upper-half plane $\omega \geq 0$ is displayed.

Poincaré waves, characterized, respectively, by the inequalities

$$
\begin{aligned}
& \omega^{2} \leq \frac{1+2 n}{2}-[n(n+1)]^{1 / 2} \text { and } \\
& \omega^{2} \geq \frac{1+2 n}{2}+[n(n+1)]^{1 / 2} .
\end{aligned}
$$

There is a wide-scale separation between the Rossby and the Poincaré waves.

\section{b. Resonances between long waves through topography}

Since the dispersion relations for equatorially trapped waves (shown in Fig. 1) are far from self-similar, the nature of resonant interactions depends strongly on the order of magnitude of the wavenumbers in play. The focus here is on very long waves, with spatial periods comparable to the circumference of the earth, $P=$ $40000 \mathrm{~km}$.

A Kelvin wave $K(x-t)$ can be written as a Fourier series

$$
K(x-t)=\sum_{l=1}^{\infty} \hat{K}(l) e^{i \alpha l(x-t)},
$$

where $\alpha=(2 \pi L) / P$ and $L=(c / \beta)^{1 / 2}$ is the equatorial length scale. A wave with $l=1$, that is, with period equal to the circumference of the earth, has a wavenumber $k=\alpha$, and all other wavenumbers are integer multiples of this. Similarly, each mode $D_{j}$ of the dispersive waves in (2.7)-(2.9) has the form

$$
D(x, t)=D_{j} e^{i\left(\alpha j x-\omega_{j} t\right)},
$$


TABLE 1. Typical speeds, and corresponding units of length, time, and wavenumber.

\begin{tabular}{rccc}
\hline \hline$c\left(\mathrm{~m} \mathrm{~s}^{-1}\right)$ & $L(\mathrm{~km})$ & $T(\mathrm{~h})$ & $\alpha$ \\
\hline 50 & 1500 & 8 & 0.23 \\
100 & 2100 & 6 & 0.33 \\
300 & 3600 & 3 & 0.57 \\
\hline
\end{tabular}

where $j$ is an integer and $\omega_{j}=W(j \alpha)$. Table 1 displays approximate values of $L, T$, and $\alpha$ for typical values of $c$.

As a Kelvin wave interacts with the topography, it can generate other waves through a three-mode resonance. If $k_{K}, k_{T}$, and $k^{*}$ denote the wavenumbers of the Kelvin wave, the topography, and the dispersive wave, respectively, the conditions for resonance are

$$
\begin{aligned}
k_{K}+k_{T} & =k^{*} \quad \text { and } \\
k_{K} & =\omega^{*}=W\left(k^{*}\right),
\end{aligned}
$$

where for the Kelvin wave $\omega=k$ and the topography is time independent. The resonant interaction conditions in (2.11) and (2.12) are derived from the detailed asymptotic derivation in section 5. Since all the wavenumbers have to be multiples of $\alpha$, the nature of the possible interactions depends on the numerical value of $\alpha$. In the next two subsections, two typical cases corresponding to the barotropic and first baroclinic modes of the tropical atmosphere are studied. The barotropic and first baroclinic modes have wave speeds given approximately by $c=300 \mathrm{~m} \mathrm{~s}^{-1}$ and $c=50 \mathrm{~m} \mathrm{~s}^{-1}$, respectively (Milliff and Madden 1996). Here a value of $c=50 \mathrm{~m} \mathrm{~s}^{-1}$ is utilized to represent the typical wave speed associated with the response to diabatic heating from convection (Gill 1982; Yano et al. 1995; Milliff and Madden 1996). This is partly justified by the discussion in section $5 \mathrm{~b}$ below regarding the nonlinear response to diabatic heating. The value of $c \cong 150 \mathrm{~m} \mathrm{~s}^{-1}$ is actually more appropriate for the first baroclinic mode of the dry atmosphere including both the troposphere and stratosphere (Kasahara and Puri 1981). This example is not discussed in this paper for brevity.

\section{c. The barotropic mode}

Since $c$ is approximately $300 \mathrm{~m} \mathrm{~s}^{-1}$, a typical value for the barotropic mode, $\alpha$, is close to 0.57. In Fig. 2, we have redrawn the dispersion relations of Fig. 1, focusing on the longest waves and displaying the grid corresponding to allowable wavenumbers and (Kelvin) frequencies for this value of $\alpha$. Which large-scale dispersive waves can resonate with a Kelvin wave via topography and satisfy (2.11) and (2.12)? Since the Kelvin wave cannot have a positive wavenumber $k_{K}$ smaller than $\alpha>0.3$, from inspection of the dispersion relation in Fig. 2, it follows that no Rossby waves can result from the interaction of a Kelvin wave with topography, and the only wave that can be generated by the first two modes of a Kelvin wave is the $k=-1.14(l=-2)$

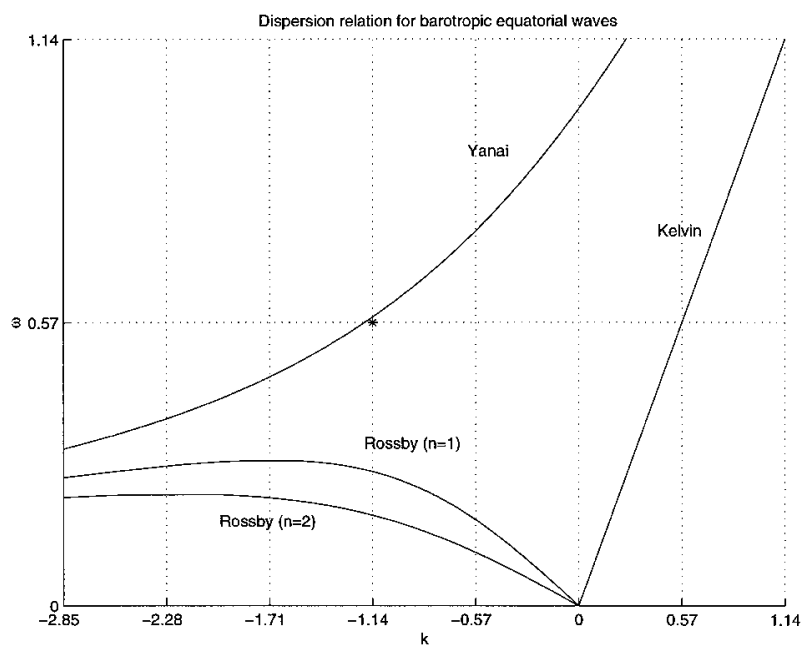

FIG. 2. Dispersion relation for equatorial waves that are concentrated on the barotropic mode. The grid displayed corresponds to allowable wavenumbers, which follows from measuring the circumference of the earth in the dimensional units corresponding to the barotropic mode. The asterisk denotes the Yanai mode $(k=-2$ in units of $\alpha$ ) that resonates with the $k=1$ mode of the Kelvin wave through the $k=3$ mode of the topography.

Yanai mode, generated by the $l=1$ Kelvin wave, and the $l=3$ mode of the topography.

From the above discussion, reduced dynamic equations for small-amplitude waves and topography should emerge involving the Kelvin modes and the large-scale Yanai wave. Such an asymptotic expansion is carried out in detail in section 5 . The resulting equations are

$$
\begin{aligned}
K_{\tau}+\left(\frac{1}{2} K^{2}\right)_{\theta} & =Y(\tau) e^{i \theta}+\bar{Y}(\tau) e^{-i \theta} \text { and } \\
Y_{\tau} & =-\hat{K}(1, \tau),
\end{aligned}
$$

where $K(\theta, \tau)$ and $Y(\tau)$ stand for the Kelvin wave and the $l=-2$ mode of Yanai, respectively, $\theta=\alpha(x-t)$ represents the linear phase of the Kelvin wave (normalized so that it has period $2 \pi$ ) and $\tau=\epsilon t$ represents the slow nonlinear time (where $\epsilon \ll 1$ is a nondimensional measure of the amplitude of the topographic variations). The dependent variables $K$ and $Y$ and the slow time $\tau$ have been further rescaled in order to normalize to one the interaction coefficients on the right-hand side, which depend on the projection of the (zonal) mode $l$ $=3$ of the topography on the (longitudinal) first Hermite polynomial [see (5.33) and (5.34) below].

Equation (2.13) for the evolution of the Kelvin wave is an inviscid Burgers equation, forced on the right-hand side by the interaction of the Yanai wave with the topography. The Burgers equation arises because Kelvin waves are nondispersive, so all modes resonate with each other. Equation (2.14), on the other hand, is an ordinary differential equation for the evolution of the Yanai wave, forced by the interaction of the Kelvin wave with the topography. This system of equations has very 
distinctive properties, some of which are treated below in section 3 .

\section{d. The first baroclinic mode (multiple wave interaction)}

The first baroclinic mode associated with convective heating typically has a characteristic speed close to 50 $\mathrm{m} \mathrm{s}^{-1}$, with values of $\alpha$ around 0.25 . Figure 3 shows the corresponding blowup of the dispersion relation. More interactions can take place in this case through the topographic resonance mechanism in (2.11) and (2.12) than in the barotropic situation described above: the $l=1(k=0.25)$ mode of the Kelvin wave can interact with the $l=4(k=-1)$ mode of the first Rossby wave through the $l=5$ mode of the topography, and the $l=2$ and $l=4$ Kelvin modes can interact with the $l=6(k=-1.5)$ and $l=0$ Yanai modes, respectively, through the $l=8$ and $l=4$ modes of the topography. The $l=12$ mode of the first Rossby wave is also formally in resonance with the $l=1$ Kelvin wave but requires a significant amplitude of a short wavelength $(l=13)$ of the topography, which is assumed to be zero for simplicity here.

Thus, only the three resonances mentioned above are considered here. The asymptotic procedure for small amplitude waves and topography from section 5 for this case yields the following set of reduced equations:

$$
\begin{aligned}
K_{\tau}+\left(\frac{1}{2} K^{2}\right)_{\theta}= & \gamma_{1}\left(R(\tau) e^{i \theta}+\bar{R}(\tau) e^{-i \theta}\right) \\
& +\gamma_{2}\left(Y_{6}(\tau) e^{2 i \theta}+\bar{Y}_{6}(\tau) e^{-2 i \theta}\right) \\
& +\gamma_{4}\left(Y_{0}(\tau) e^{4 i \theta}+\bar{Y}_{0}(\tau) e^{-4 i \theta}\right), \\
R_{\tau}= & -\gamma_{1} \hat{K}(1, \tau), \quad Y_{6 \tau}=-\gamma_{2} \hat{K}(2, \tau), \quad \text { and } \\
Y_{0 \tau}= & -\gamma_{4} \hat{K}(4, \tau) .
\end{aligned}
$$

Here the constants $\gamma_{j}$ account for the strength of the various topographic interactions.

Clearly, this system of equations represents a mild generalization of the one corresponding to the barotropic mode with added richness provided by its larger number of degrees of freedom.

\section{Properties of the model dynamics}

The reduced models discussed in sections $2 \mathrm{c}$ and $2 \mathrm{~d}$ for large-scale waves interacting with Kelvin waves via topographic resonance have a number of distinctive features. Some of these properties and their physical implications are developed in this section. In section 3a, the nature of the quantities conserved by the system are discussed. In section $3 b$, an explicit family of traveling wave solutions is constructed; which indicates that the large-scale dispersion in (2.13), (2.14), or (2.15) can prevent the breaking of Kelvin waves and significantly

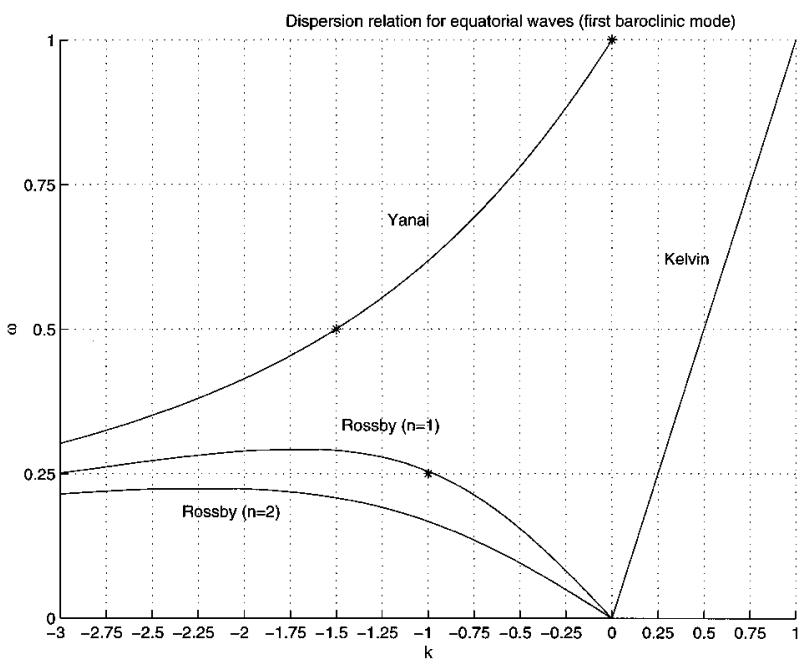

FIG. 3. Dispersion relation for equatorial waves with a grid corresponding to the first baroclinic mode. In this case, there are three dispersive modes resonating with the Kelvin wave through the topography.

modify (slow down) their speed. In sections $3 \mathrm{c}$ and $3 \mathrm{~d}$, a series of numerical experiments are presented, which show that these traveling wave solutions strongly attract many initial data with enough energy and that there is a larger class of attracting solutions, which are oscillatory with two periods in time (quasiperiodic). This situation is similar to that arising the in context of gas dynamics, when left- and right-going waves interact through a variable entropy (Shefter 1997). For clarity in presentation, the barotropic case with only two waves is discussed first and then the extra ingredients from multiple wave interactions. The latter is done with the general system

$$
\begin{aligned}
K_{\tau}+\left(\frac{1}{2} K^{2}\right)_{\theta} & =\sum_{j=1}^{m} \gamma_{j}\left[R_{j}(\tau) e^{i j \theta}+\bar{R}_{j}(\tau) e^{-i j \theta}\right] \quad \text { and } \\
R_{j_{\tau}} & =-\gamma_{j} \hat{K}(j, \tau),
\end{aligned}
$$

with the $\gamma_{j}$ real, which includes the baroclinic equations in (2.15) as a particular case.

\section{a. Conserved quantities and symmetries}

The equations in (2.13) and (2.14) have two main conserved quantities, the mean of $K$ and the total energy. Conservation of the mean follows straightforwardly from integrating (2.13) along a period; it takes the form

$$
\frac{d}{d \tau} \int_{-\pi}^{\pi} K(\theta, \tau) d \theta=0 .
$$

In particular, this equation tells us that it is sufficient to consider solutions with constant zero mean since a nonzero mean can be absorbed into the unperturbed depth of the fluid layer. 
To see the form for conservation of energy, multiply Eq. (2.13) by $K$ and integrate

$$
\begin{aligned}
\frac{d}{d t} \int_{-\pi}^{\pi} \frac{1}{2} K^{2}(\theta, \tau) d \theta & =2 \pi[Y(\tau) \hat{K}(-1, \tau)+\bar{Y}(\tau) \hat{K}(1, \tau)] \\
& =2 \pi(Y(\tau) \hat{K}(1, \tau)+\bar{Y}(\tau) \hat{K}(1, \tau)) \\
& =-2 \pi\left(|Y|^{2}\right)_{\tau},
\end{aligned}
$$

so the energy

$$
E=\int_{0}^{2 \pi} \frac{K^{2}}{2} d \theta+2 \pi|Y|^{2}
$$

is conserved while the solution remains smooth. However, energy may be dissipated at locations where the Kelvin wave breaks (Majda et al. 1988). Here the detailed physical dissipative and dispersive mechanisms for breaking Kelvin waves are not developed since, as shown below, the equations allow robust traveling wave solutions without discontinuities.

The equations in (2.13) and (2.14) have an interesting symmetry: they remain invariant under the transformation

$$
K \rightarrow-K, \quad Y \rightarrow \bar{Y}, \text { and } \quad \theta \rightarrow-\theta .
$$

This symmetry is useful for the study of the energypreserving waves, as discussed in the remainder of this section.

For the more general multiwave system in (3.1), (3.2), with (2.15) as a special case, the equation in (3.3) remains unchanged, while the conserved energy becomes

$$
E=\int_{0}^{2 \pi} \frac{K^{2}}{2} d \theta+2 \pi \sum_{j=1}^{m}\left|R_{j}\right|^{2} .
$$

The symmetry described above also remains valid, with $Y$ replaced by $R_{j}$.

\section{b. Traveling wave solutions}

The equations in (2.13) and (2.14) have a family of exact solutions where $K$ is a traveling wave. To see this, an ansatz is utilized in which

$$
K(\theta, \tau)=F(\theta-s \tau),
$$

where $s$ is an arbitrary constant. The quantity $s$ represents the correction to the linear wave speed for the Kelvin wave. The Fourier transform of $K$ takes the form

$$
\hat{K}_{1}=\hat{F}_{1} e^{-i s \tau}
$$

so the equation for $Y$ becomes

$$
Y_{\tau}=-\hat{F}_{1} e^{-i s \tau},
$$

with the particular solution

$$
Y(\tau)=\frac{\hat{F}_{1}}{i s} e^{-i s \tau} .
$$

Thus Eq. (2.13) becomes the ODE.

$$
[-s+F(z)] F^{\prime}(z)=\frac{\hat{F}_{1}}{i s} e^{i z}+\frac{\overline{\hat{F}}_{1}}{i s} e^{-i z}=\frac{2 \hat{F}_{1}}{s} \sin (z),
$$

where for convenience the origin for the phase $z=\theta$ $-s \tau$ has been selected in such a way that $F(z)$ is even and $\hat{F}_{1}$ is correspondingly real. With the variable

$$
g(z)=F(z)-s,
$$

the equation in (3.7) becomes

$$
\left(\frac{g^{2}}{2}\right)^{\prime}=\frac{2 \hat{F}_{1}}{s} \sin (z)
$$

with the solution

$$
g(z)= \pm 2\left(-\frac{\hat{F}_{1}}{s}\right)^{1 / 2}[C+\cos (z)]^{1 / 2},
$$

where $C \geq 1$ is a constant of integration. From this calculation it follows that

$$
F(z)=s \pm 2\left(-\frac{\hat{F}_{1}}{s}\right)^{1 / 2}[C+\cos (z)]^{1 / 2} .
$$

Notice that if $C$ is strictly larger than one, the solution is smooth, but when $C=1$, it develops a corner. In the latter case, the solution is

$$
F(z)=s \pm 2\left(-\frac{2 \hat{F}_{1}}{s}\right)^{1 / 2}|\cos (z / 2)| .
$$

In both situations, the nonlinear breaking of the Kelvin waves has been suppressed and from (3.6) and (3.9), the wave speed has been altered through topographic resonance.

The value of $s$ follows from equating the first Fourier mode of the solution to $\hat{F}_{1}$, and imposing the requirement that $F$ have zero mean. For the interesting case with a corner, it follows that

$$
\begin{aligned}
\hat{F}_{1} & =\frac{1}{\pi} \int_{0}^{\pi} F(z) \cos (z) d z \\
& = \pm \frac{2}{\pi}\left(-\frac{2 \hat{F}_{1}}{s}\right)^{1 / 2} \int_{0}^{\pi} \cos (z / 2) \cos (z) d z \\
& = \pm \frac{4}{3 \pi}\left(-\frac{2 \hat{F}_{1}}{s}\right)^{1 / 2},
\end{aligned}
$$

So

$$
\hat{F}_{1}=-\frac{32}{9 \pi^{2} s},
$$

and

$$
F(z)=s \pm \frac{16}{3 \pi|s|}|\cos (z / 2)| .
$$

For $F$ to have zero mean requires 


$$
0=\int_{-\pi}^{\pi} F(z) d z=2 \pi s \pm \frac{64}{3 \pi|s|}
$$

so that

$$
s=\mp \frac{4}{\pi} \sqrt{\frac{2}{3}} \sim \mp 1.04 .
$$

In the original equations [Eqs. (1.1) and (1.2)], this nonlinear wave speed is $s \sim \mp 1.04 \gamma$, where $\gamma$ is the topographic amplitude coefficient. Thus the wave with a downward peak moves more slowly than a linear Kelvin wave, and the one with the upward peak moves faster (notice that these two waves are related by the symmetry group presented in the previous subsection). The total energy corresponding to this exact solution is readily computed; its value is

$$
E=\frac{14 \pi}{9}-\frac{32}{3 \pi} \sim 1.5
$$

The case with $C>1$ can be evaluated similarly in terms of elliptic functions. For a qualitative result, however, these solutions are not needed explicitly; it follows directly from the condition of zero mean that the solutions with the "+" sign (which have their "peaks" pointing downward) will be slowed down by the nonlinear interaction through topographic resonance. It is interesting to note that the speed $s$ of the traveling wave does not depend strongly on its amplitude. To this end, consider a nearly linear solution, that is, with $C \gg 1$. In this case, Eq. (3.8) is well approximated by

$$
F(z) \sim s \pm 2\left(-\frac{\hat{F}_{1}}{s}\right)^{1 / 2} \sqrt{C}\left[1+\frac{\cos (z)}{2 C}\right],
$$

so

$$
\hat{F}_{1} \sim\left(-\frac{\hat{F}_{1}}{4 s C}\right)^{1 / 2}
$$

or

$$
\hat{F}_{1} \sim-\frac{1}{4 s C}
$$

The condition for zero mean of $F(z)$ then becomes so

$$
s \sim \mp 1 \text {. }
$$

Thus the magnitude of $s$ ranges between 1.04 and 1, depending on the amplitude of the traveling wave. Since this range is very short, it is not the strength of the waves that determines the nonlinear speed of the Kelvin wave but only the amplitude of the topography (which determines $\epsilon$, the scale of the nonlinear time). This speed $s \sim \pm 1$ arises in all numerical experiments presented in section $3 \mathrm{c}$, independent of the nature of the initial data.

The more general equations in (3.1) and (3.2) admit similar traveling wave solutions with and without corners. The analysis is entirely similar to the one above: with a traveling Kelvin wave $K(\theta, \tau)=F(\theta-s \tau)$, it follows that each dispersive wave $R_{j}(\tau)$ oscillates with frequency $j s$. The general form of $F(z)$ is

$$
F(z)=\frac{s}{2} \pm[C+H(z)]^{1 / 2},
$$

where $H(z)$ is a real periodic function with $j$ th Fourier coefficient

$$
\hat{H}_{j}=-\frac{\gamma_{j} \hat{F}_{j}}{j^{2} s}
$$

for $j$ positive and $C \geq \max (-H)$ is an arbitrary constant. When $C=\max (-H)$, the resulting traveling wave has at least one corner; again the solutions with corners facing downward move more slowly than a linear Kelvin wave.

A difference between this general case and the one with a single dispersive wave, is that the speed of the traveling wave is not constrained to be close to \pm 1 . A way to see this is to consider the linearized equations,

$$
\begin{aligned}
& K_{\tau}=\sum_{j=1}^{m} \gamma_{j}\left[R_{j}(\tau) e^{i j \theta}+\bar{R}_{j}(\tau) e^{-i j \theta}\right] \text { and } \\
& R_{j_{\tau}}=-\gamma_{j} \hat{K}(j, \tau),
\end{aligned}
$$

and their family of solutions,

$$
K=\sum_{j=1}^{m} \alpha_{j} \cos \left(\theta \mp \gamma_{j} \tau\right) \quad \text { and } \quad R_{j}= \pm \frac{1}{2} \alpha_{j} e^{-i \gamma_{j} \tau},
$$

where each mode moves independently at speed $\pm \gamma_{j}$. Thus in the nonlinear traveling waves, a range of speeds should emerge, depending on the strength of the dominant topographic modes, $\gamma_{j}$.

\section{c. Numerical experiments}

One might wonder about the significance of the traveling wave solutions of the previous subsection: Do general initial data tend toward these solutions? If not, is the behavior of more general solutions in any way similar to that of the traveling waves? In this section, these questions are addressed through a few selected numerical experiments.

Solving numerically the Eqs. (2.13) and (2.14), or the more general (3.1) and (3.2), is a relatively straightforward task. Here a fractional step procedure is utilized, solving in one step the inviscid Burgers equation,

$$
K_{\tau}+\left(\frac{K^{2}}{2}\right)_{\theta}=0
$$

and in the other the system of integro-differential equations

$$
\begin{aligned}
& K_{\tau}=\sum_{j=1}^{m} \gamma_{j}\left[R_{j}(\tau) e^{i j \theta}+\bar{R}_{j}(\tau) e^{-i j \theta}\right] \quad \text { and } \\
& R_{j_{\tau}}=-\gamma_{j} \hat{K}(j, \tau)
\end{aligned}
$$


For the inviscid Burgers equation, a second-order Godunov method is used, and the system of integro-differential equations is integrated with a second-order Runge-Kutta method coupled with a fast Fourier transform. Finally, the two fractional steps are put together using the second-order procedure of Strang (1968). A Godunov scheme is utilized to treat the breaking Kelvin wave solutions that might evolve and dissipate energy in a conservative fashion. We do not view this dissipative mechanism for breaking Kelvin waves as the physically correct one for modeling equatorial waves; instead, we regard it as a convenient numerical device to reveal the robustness of the traveling waves without discontinuities described in section $3 \mathrm{~b}$. We speculate that breaking Kelvin waves could radiate energy into families of nonresonant modes.

The reduction of complexity in going from the full system in (2.1), (2.2), and (2.3) to the reduced model (3.1) and (3.2) is so big that the model is programmed in the interpreter language Matlab with a typical run with around 100 grid points taking less than a minute on a workstation. Calculations with far more points were performed here to obtain greater resolution. Such calculations would have been extremely costly for the full system. Next the results of four typical experiments are presented, three for the barotropic, and one for the first baroclinic mode.

\section{THE BAROTROPIC MODE}

The first experiment illustrates quite dramatically the "attractive" nature of the traveling wave solution in (3.9). Figure $4 \mathrm{a}$ shows the initial value of $K$, given by an arbitrarily chosen periodic function; in this case,

$$
K(\theta, 0)=0.5[3 \cos (\theta)+\sin (2 \theta)-\sin (4 \theta)],
$$

the initial value assigned to $Y(0)$ is

$$
Y(0)=0.5(1+i) .
$$

The total initial energy of this solution is $E=7.5$, which is larger than the threshold value 1.5 reported above in (3.11) for the traveling Kelvin wave with a corner (the solution with a corner has the largest energy among traveling waves). In Fig. 4b, we see the solution $K$ at $\tau=0.5$, with a freshly created strong breaking Kelvin wave. By the time $\tau=5$, displayed in Fig. 4c, most of the extra energy of the initial data has been dissipated through the breaking wave, which has also eliminated all but the longest modes of the solution. Finally, by the time $\tau \sim 501$ as in Fig. 4d, a steady state is approached. This state agrees nearly exactly with the exact traveling wave with a corner in (3.9), which is also displayed.

Figure 5a shows the time evolution of the real and imaginary part of $Y(\tau)$, the amplitude of the Yanai wave. Notice that, since the speed $s$ of the traveling Kelvin wave is close to one, and this equals the frequency of the Yanai wave, the period of oscillation of the latter is close to $2 \pi$. Finally, Fig. $5 \mathrm{~b}$ has the total energy as a function of time, showing the fast initial dissipation due to Kelvin wave breaking, followed by stabilization at nearly the exact critical energy corresponding to the wave with a sharp corner.

Something entirely similar takes place when the initial energy is already below the threshold value: rather than converging to one of the smooth traveling wave solutions in (3.8) with $C>1$, most initial data converge, after some initial energy dissipation through Kelvin wave breaking, to a quasi-periodic pattern qualitatively similar to a traveling wave. As an example, the case is solved numerically with initial conditions $K(\theta, 0)=0.1$ $[3 \cos (\theta)+5 \sin (2 \theta)-\sin (4 \theta)], Y(0)=0.1(1+i)$, with total energy $E=0.675$, much below the critical $E=1.5$. From Fig. 6a, this total energy decays to a final value close to $E=0.123$. However, as shown in Fig. 7, this final state is not strictly a traveling wave, but a quasi-periodic wave, with period close to $2 \pi$. Snapshots of $K$ for four times over a period are depicted: even though the wave does travel to the left at a speed close to 1 , it deforms as it does so, developing along the way a corner similar to those of the solution with maximal energy. Figure $6 \mathrm{~b}$, with the evolution of the Yanai wave, confirms that the solution is indeed quasiperiodic and not strictly periodic, as there is a slow periodic modulation of the oscillations of the real and imaginary part of the wave's amplitude.

One may wonder why a nontrivial combination of the $s=+1$ and $s=-1$ waves never appears in a quasisteady solution. A partial answer is provided by the following example: consider initial data such that $K(\theta, 0)$ is odd and $Y(0)$ is purely imaginary. Such initial data is invariant under the symmetry described in section $3 \mathrm{a}$, so any wave with speed +1 must necessarily be accompanied by one with speed -1 . Figures $8 \mathrm{a}$ and $8 \mathrm{~b}$ show the evolution of the initial data $K(\theta, 0)=\sin (\theta)$, $Y(0)=0.5 i$. In Fig. 8a, we see that, unlike the previous experiments, the energy decays continuously, without stabilizing as before at some positive value-it is apparent that the imposed symmetry between right- and left-going waves excludes all energy preserving solutions. The energy-dissipating breaking Kelvin waves become the most prominent part of the solution as it decays to zero. Figure $8 \mathrm{~b}$ shows the evolution of the accompanying imaginary part of $Y(\tau)$ (its real part is zero), which clearly displays decay.

\section{d. The baroclinic mode: Multiple waves}

The baroclinic case, with multiple wave interactions, is richer than the barotropic one with only one dispersive wave. Nevertheless, its solutions are generically not very different from those with one single resonance because the largest wavelength of the problem tends to prevail, thus selecting one preferred dispersive wave, with the others decaying to much smaller levels.

The experiment displayed in Figs. 9a-d illustrates this 
(a)

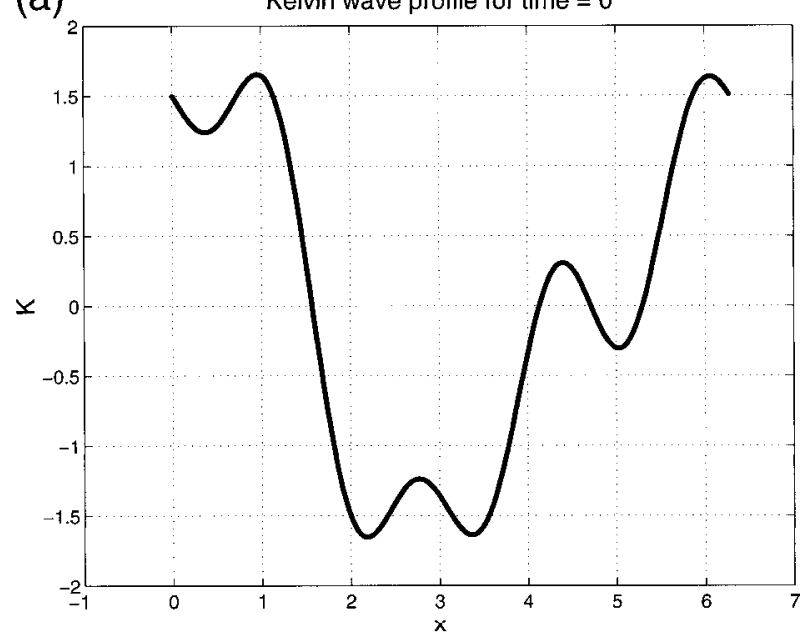

(c)

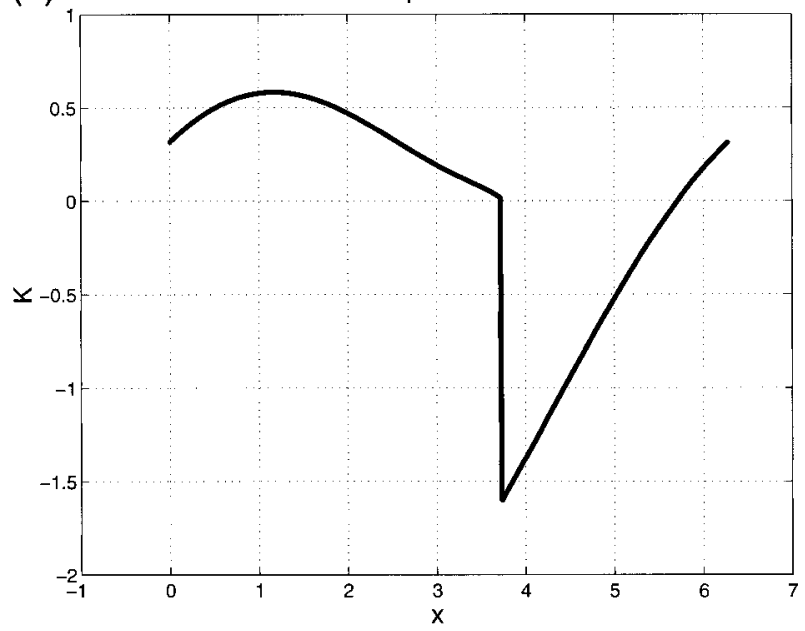

(b)

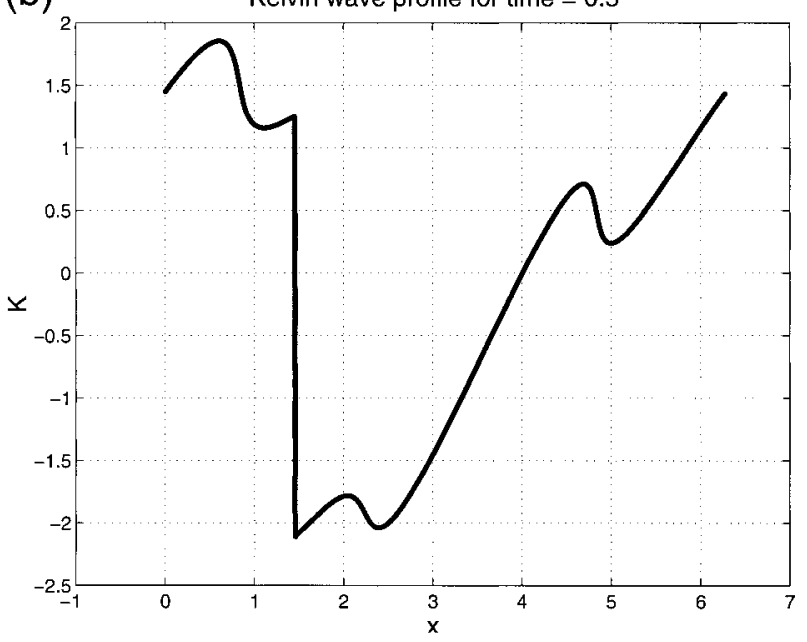

(d)

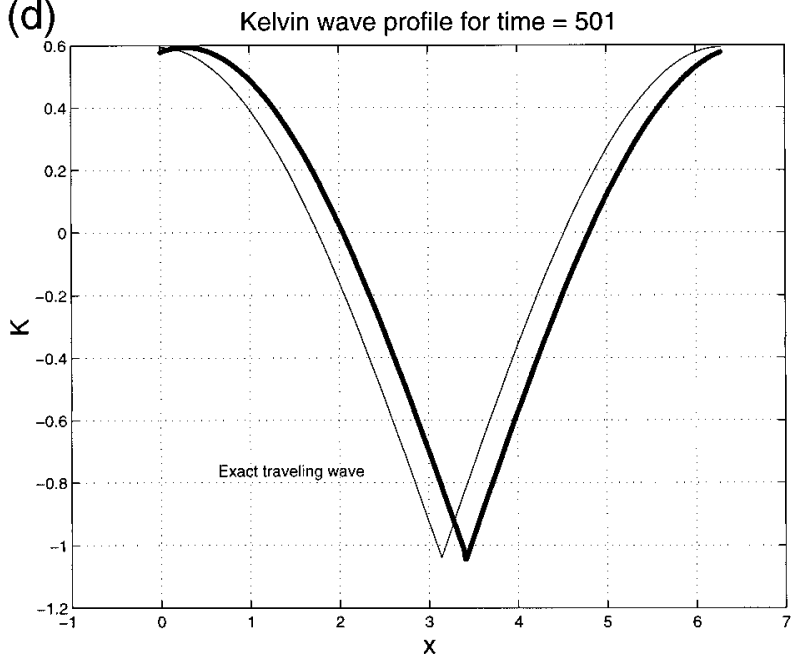

FIG. 4. A numerical experiment on the barotropic mode displaying convergence to a traveling wave with a corner. (a) Initial profile of the Kelvin wave: $K(\theta, 0)=0.5[3 \cos (\theta)+\sin (2 \theta)-\sin (4 \theta)]$. (b) Profile at $\tau=0.5$ : the Kelvin wave has steepened nonlinearly and developed a sharp discontinuity. (c) By the time $\tau=5$, most of the extra energy in the initial data has dissipated at the breaking wave. (d) The Kelvin wave at $\tau=501$ has all but converged to the exact traveling wave with maximal energy. Only a tiny amount of extra energy still needs to be dissipated at an extremely small amplitude breaking wave.

phenomenon. Its setup includes the waves discussed in section 2d: a Kelvin wave, three dispersive waves, the $k=4$ mode of the $n=1$ Rossby wave, and the $k=6$ and $k=0$ modes of the Yanai wave, interacting with the $k=1, k=2$, and $k=4$ modes of the Kelvin wave through the $k=5, k=8$, and $k=4$ modes of the topography, respectively. To prevent bias in the experiment, the topographic interaction coefficients $\gamma_{j}$ have been chosen close to one, with values $1,0.9$, and 0.8 , respectively, and the initial data has the three dispersive waves with comparable amplitudes; that is,

$$
\begin{aligned}
K(x, 0) & =0.3[3 \cos (x)+7 \sin (2 x)-3 \sin (4 x)] \text { and } \\
R_{4}(0) & =Y_{6}(0)=Y_{0}(0)=0.3(1+i) .
\end{aligned}
$$

Figure 9a shows that, as before, the energy decays very rapidly to a final nonzero level where it settles down. Figure $9 \mathrm{~b}$ shows that only the Rossby wave with wavenumber $n=4$, which interacts with the Kelvin mode with largest wavelength (i.e., $2 \pi$ in the normalized variables), has a significant component in the final, quasi-periodic state. This is further confirmed by the snapshots of the Kelvin wave, displayed in Fig. 9c, with a quasi-periodic pattern very similar to those of the barotropic case, and with only small amplitude wiggles in the larger frequencies.

\section{Effects of the mean wind}

The effects of an externally imposed, uniform mean wind are easily incorporated into the model dynamics. 
(a)
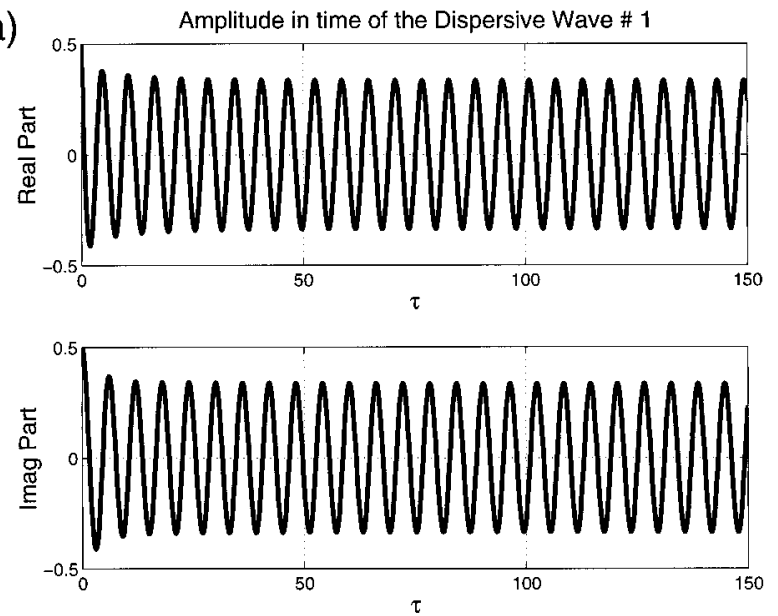

(b)

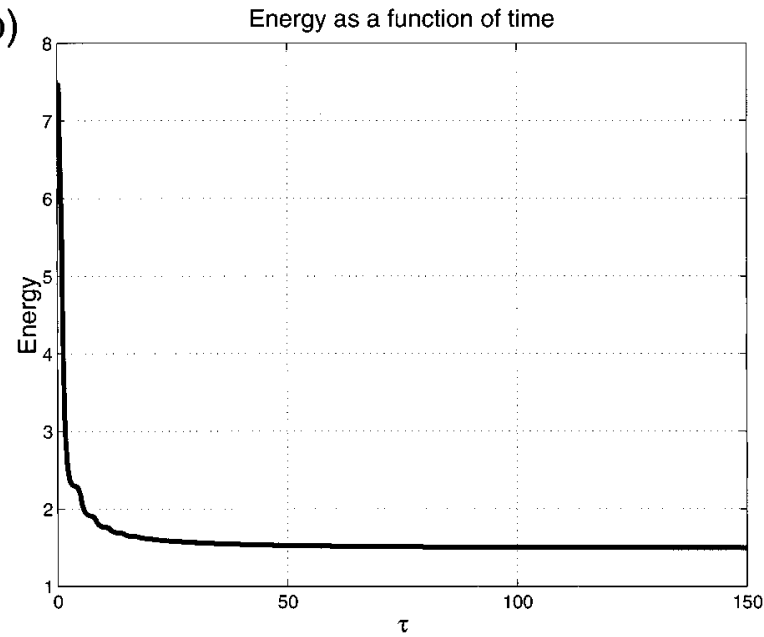

FIG. 5. (a) Time evolution of the amplitude of the Yanai wave for the numerical experiment above. It soon converges to a periodic oscillation with a period close to $2 \pi$. (b) The total energy decays very rapidly initially, and then very slowly, converging to the value corresponding to the exact traveling wave.

Of course, the tacit hypothesis in assuming a uniform mean wind is that this imposed velocity is both wider and deeper than the structure of the waves being investigated. Such a requirement can be invalid in many physical circumstances; nevertheless, this assumption is made here to illustrate some potentially interesting ramifications of the topographic resonant theory. To search for resonances through topography, it is convenient to leave the topography fixed, so that its dispersion relation has $\omega=0$ as before, and consider instead the Doppler effect of the mean wind on the dispersion relation for the waves, which becomes

$$
W(k)=W^{*}(k)+U k,
$$

where $W^{*}(k)$ is the dispersion relation without mean wind and $U$ is the velocity of the wind, measured using the characteristic speed $c$ as a unit. With (4.1), the con- (a)

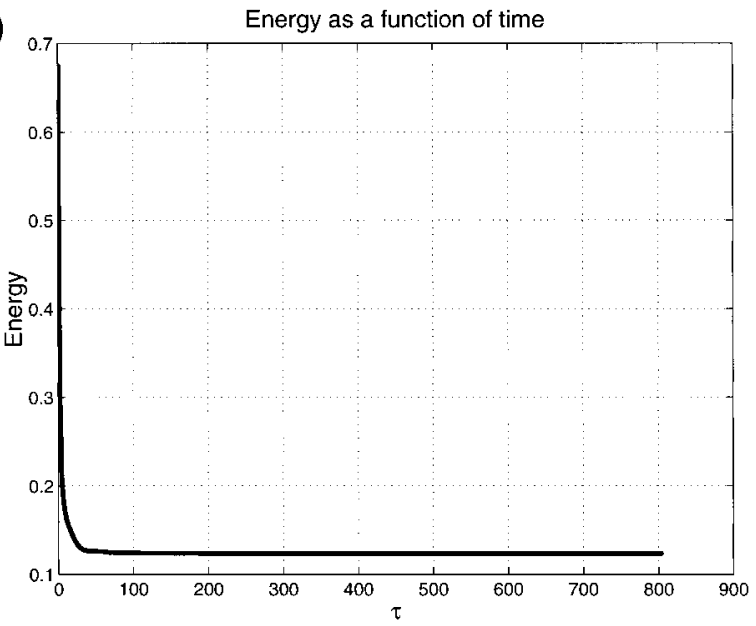

(b)
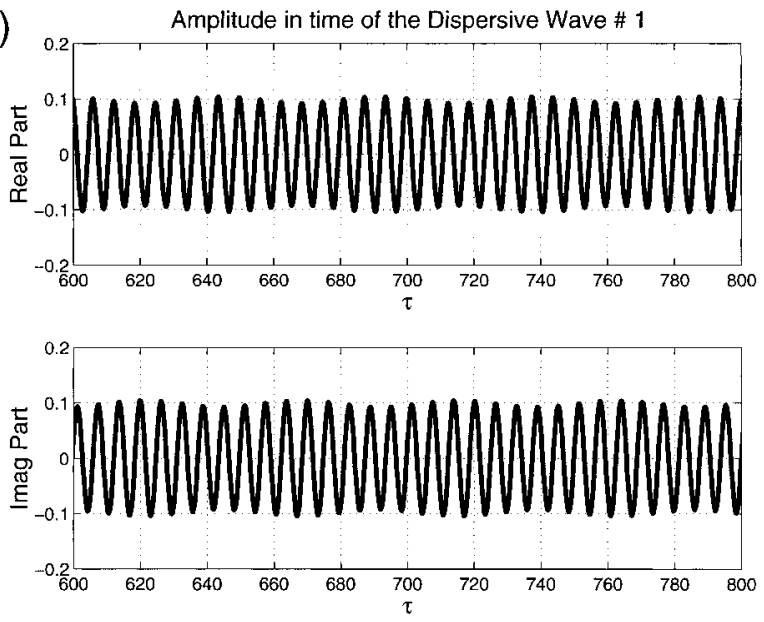

FIG. 6. A second experiment on the barotropic mode, this one with a smaller initial energy. The solution converges to a quasi-periodic wave with finite amplitude. (a) Energy as a function of time, showing fast initial decay and subsequent stabilization at a nonzero value. (b) Evolution of the amplitude of the Yanai wave. A periodic slow modulation of the oscillations clearly appears. For clarity, only the times between $\tau=600$ and $\tau=800$ are displayed.

ditions in (2.11) and (2.12) for topographic resonance with a mean wind $U$ generalize to

$$
k_{K}+k_{T}=k^{*} \quad \text { and } \quad k_{K}+V k_{K}=W\left(k^{*}\right)+V k^{*} \text {. }
$$

Figures 10 and 11 display the new dispersion relation for two extreme cases, with $U=c / 2$ and $U=-c / 2$, that is, for a wind moving, respectively, eastward and westward at half the speed of a linear Kelvin wave. A unit for the wavenumbers, $\alpha=0.25$, is selected corresponding to the first baroclinic mode; notice, however, that the unit of frequencies $\omega$ is different due to the distortion brought about by the Doppler effect. Figures 10 and 11 indicate that relatively strong mean winds (about $25 \mathrm{~m} \mathrm{~s}^{-1}$ ) can change the general look of the dispersion relation quite dramatically. However, it is still true that the Kelvin wave is the only nondispersive wave, and the role of topography remains the same, so 


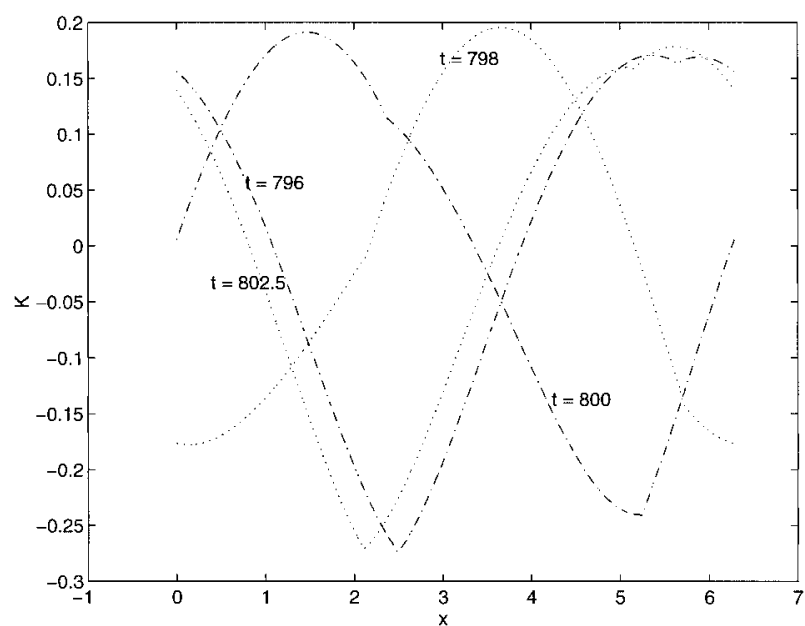

FIG. 7. Four snapshots of the Kelvin wave close to $\tau=800$. The downward peak is clearly visible, as well as the quasi-periodic character of the wave, which deforms continuously as it moves but returns periodically to the initial shape. the basic structure of our reduced model does not change, only the modes involved in the resonances do. One important feature noticeable in both Figs. 10 and 11 is that Rossby waves of all longitudinal Hermite modes are present, in what appears as a cluster of modes. The appearance of such a spectrum of oscillatory Rossby waves suggests a likely midlatitude connection of equatorial Kelvin waves through topographic resonance. This aspect, and the effects of a latitude-dependent mean wind in geostrophic balance with the pressure, will be developed elsewhere in more detail in the future.

\section{Derivation of the reduced model}

\section{a. The barotropic mode}

In this section, the simplified model in (2.13) and (2.14) for the interaction of the barotropic mode of long equatorial waves through the topography is derived. The derivation begins with the ansatz,

$$
\begin{aligned}
& \eta=\epsilon\left[K(x-t, \tau) e^{-\left(y^{2} / 2\right)}+Y(\tau) \frac{y}{k^{*}-\omega^{*}} e^{-\left(y^{2} / 2\right)} e^{i\left(k^{*} x-\omega^{*} t\right)}+\text { C.C. }\right]+\epsilon^{2} \eta^{1}(x, y, t), \\
& u=\epsilon\left[K(x-t, \tau) e^{-\left(y^{2} / 2\right)}+Y(\tau) \frac{y}{k^{*}-\omega^{*}} e^{-\left(y^{2} / 2\right)} e^{i\left(k^{*} x-\omega^{*} t\right)}+\text { C.C. }\right]+\epsilon^{2} u^{1}(x, y, t), \text { and } \\
& v=\epsilon\left[0+i Y(\tau) e^{-\left(y^{2} / 2\right)} e^{i\left(k^{*} x-\omega^{*} t\right)}+\text { C.C. }\right]+\epsilon^{2} v^{1}(x, y, t),
\end{aligned}
$$

where $\omega^{*}$ and $k^{*}$ satisfy the dispersion relation for Yanai waves:

$$
\omega^{*}\left(\omega^{*}-k^{*}\right)=1 .
$$

The slow time variable $\tau$ in (5.1), (5.2), and (5.3) is $\tau$ $=\epsilon t$. It is also assumed that the topography is also small amplitude with the form

$$
h(x, y)=\epsilon\left[\sum_{j=\alpha}^{\infty} \hat{h}_{j}(y) e^{i j x}+\text { C.C. }\right],
$$

where the sum ranges over all multiples of $\alpha$.

Inserting this ansatz into (2.1), (2.2), and (2.3) to leading order in $\epsilon$, the equations are satisfied automatically since the ansatz is the superposition of two linear solutions. The equations at order $\epsilon^{2}$ become

$$
\begin{aligned}
\eta_{t}^{1}+u_{x}^{1}+v_{y}^{1} & =A, \\
u_{t}^{1}+\eta_{x}^{1}-y v^{1} & =B, \quad \text { and } \\
v_{t}^{1}+\eta_{y}^{1}+y u^{1} & =C,
\end{aligned}
$$

where

$$
\begin{aligned}
A= & -e^{-\left(y^{2} / 2\right)} K_{\tau}-e^{-y^{2}}\left(K^{2}\right)_{\theta}-\frac{y e^{-\left(y^{2} / 2\right)}}{k^{*}-\omega^{*}} e^{i\left(k^{*} x-\omega^{*} t\right)} Y_{\tau} \\
& -\frac{i \omega^{*} y^{2} e^{-y^{2}}}{k^{*}-\omega^{*}} e^{2 i\left(k^{*} x-\omega^{*}\right)} Y^{2} \\
& -\frac{y e^{-y^{2}}}{k^{*}-w^{*}}\left[K_{\theta} Y+i\left(2 \omega^{*}-k^{*}\right) K Y\right] \\
& +e^{-\left(y^{2} / 2\right)}\left(K_{\theta} h+K h_{x}\right)+\frac{y e^{-\left(y^{2} / 2\right)}}{k^{*}-\omega^{*}}\left(h_{x}+i \omega^{*} h\right) Y \\
& +i e^{-\left(y^{2} / 2\right)} h_{y} Y
\end{aligned}
$$

and $B$ and $C$ have similar expressions. The equations for the evolution in $\tau$ of the $K(x-t, \tau)$ and $Y(\tau)$ in the ansatz will follow from the solvability conditions for the system (5.6), (5.7), and (5.8).

\section{1) Evolution of the Kelvin wave}

To find the solvability condition associated with the Kelvin wave, we add Eqs. (5.6) and (5.7), multiply this 
(a)
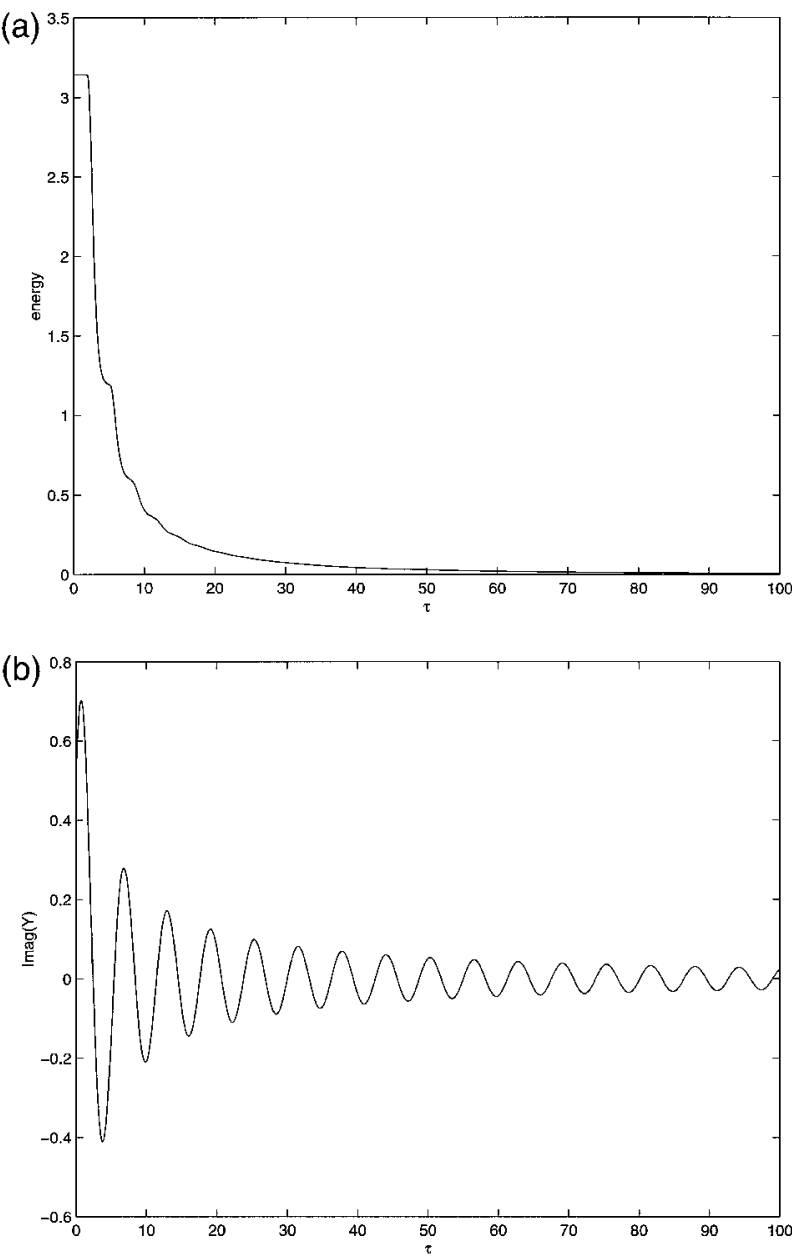

FIG. 8. A numerical experiment on the barotropic mode showing that upward and downward corners cannot coexist. This experiment exploits a symmetry of the equations that makes a one-to-one correspondence between downward and upward corners. When this symmetry is reflected in the initial data, the waves break and decay all the way to zero. (a) Energy as a function of time showing continuous decay. (b) Amplitude of the imaginary part of the Yanai wave (the real part is identically zero) with decaying oscillations.

sum by the factor $e^{y^{-2} / 2}$ and integrate in $y$ between $-\infty$ and $\infty$. The terms with $v^{1}$ are eliminated by this integration, and the equation

$$
R_{t}+R_{x}=\int_{-\infty}^{\infty} e^{-\left(y^{2} / 2\right)}(A+B) d y
$$

is obtained where

$$
R=\int_{-\infty}^{\infty} e^{-\left(y^{2} / 2\right)}\left(u^{1}+\eta^{1}\right) d y
$$

The solvability condition for this equation requires that the right-hand side of (5.10) has no component depending on $(x-t)$ since such a component-a solution to the unforced equation for $R$-would make the latter grow secularly in time. To determine which terms contribute to this secular growth, the Kelvin wave $K(x-t, \tau)$ and the topography $h(x, y)$ from (5.5) are expanded in Fourier series

$$
K(x-t, \tau)=\sum_{l=\alpha}^{\infty} \hat{K}_{l}(\tau) e^{i l(x-t)},
$$

with the sum taken over all multiples of $\alpha$. This infinite sum, and not just the finite set of waves that resonants through the topography, is needed for the Kelvin wave because this wave satisfies a dispersion relation, which makes every horizontal wavenumber resonate with all others (linear Kelvin waves are not dispersive).

The sum $A+B$ will have terms arising from different combinations of $K(x-t, \tau), Y(\tau) e^{i\left(k^{*} x-\omega^{*} t\right)}$, and $h(x, y)$. These different terms are considered separately.

- Terms linear in $K$. There is only one such term; that is,

$$
2 \int_{-\infty}^{\infty} e^{-y^{2}} K_{\tau}(x-t, \tau) d y
$$

This term is a function of $(x-t)$, so it contributes to the resonance.

- Terms linear in $Y$. These terms have no component in $(x-t)$ since $\omega^{*} \neq k^{*}$ for a Yanai wave.

- Terms quadratic in $K$. These terms are functions of ( $x$ $-t$ ), so they add to the secularity. Their sum is

$$
\frac{3}{2} \int_{-\infty}^{\infty} e^{-\left(3 y^{2} / 2\right)}\left(K^{2}\right)_{x}(x-t, \tau) d y .
$$

- Products of $K$ and $Y$. These terms do not contribute to the resonance since a typical term has the form $e^{i\left[\left(l+k^{*}\right) x-\left(l+\omega^{*}\right) t\right]}$ and $l+k^{*} \neq l+\omega^{*}$.

- Terms quadratic in $Y$. These do not contribute either since $2 k^{*} \neq 2 \omega^{*}$ for Yanai waves.

- Products of $h$ and $K$. These terms do not contribute to the resonance since $j+l \neq l$.

- Products of $h$ and $Y$. There is one term of this form, and it has a component in $(x-t)$ whenever $j+k^{*}$ $=\omega^{*}$. This is precisely the resonance condition between a Kelvin and a Yanai wave through the topography considered in (2.11) and (2.12). The term is

$$
\int_{-\infty}^{\infty}\left(\left\{e^{-y^{2}} \frac{i \omega^{*} y}{k^{*}-\omega^{*}}\left[\hat{h}_{\omega^{*}-k^{*}}(y) Y(\tau)\right] e^{i \omega^{*}(x-t)}\right\}+\text { C.C. }\right) d y .
$$

Adding up all the contributions to resonance above and equating their sum to zero, we obtain

$$
c_{1} K_{\tau}+c_{2}\left(K^{2}\right)_{\theta}=c_{3} Y(\tau) e^{i \omega^{*} \theta}+\text { C.C., }
$$

where $\theta=x-t$ and

$$
\begin{aligned}
& c_{1}=2 \int_{-\infty}^{\infty} e^{-y^{2}} d y, \quad c_{2}=\int_{-\infty}^{\infty} \frac{3}{2} e^{-\left(3 y^{2} / 2\right)} d y, \quad \text { and } \\
& c_{3}=\int_{-\infty}^{\infty} e^{-y^{2}} \frac{i \omega^{*} y}{k^{*}-\omega^{*}} \hat{h}_{\omega^{*}-k^{*}}(y) d y .
\end{aligned}
$$


(a)

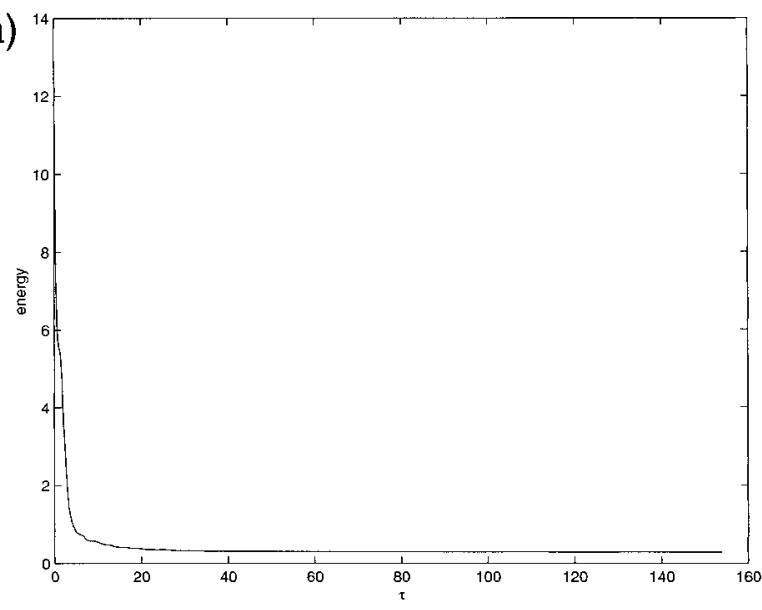

(c)

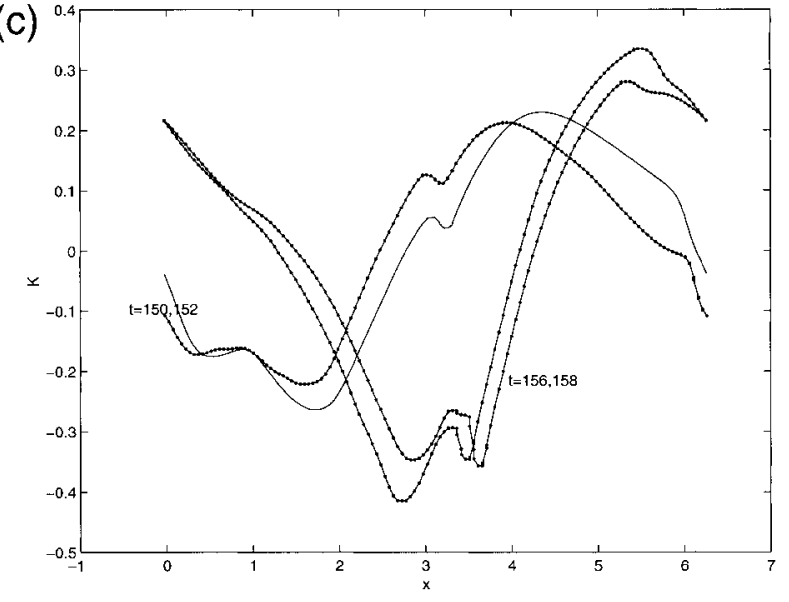

(b)
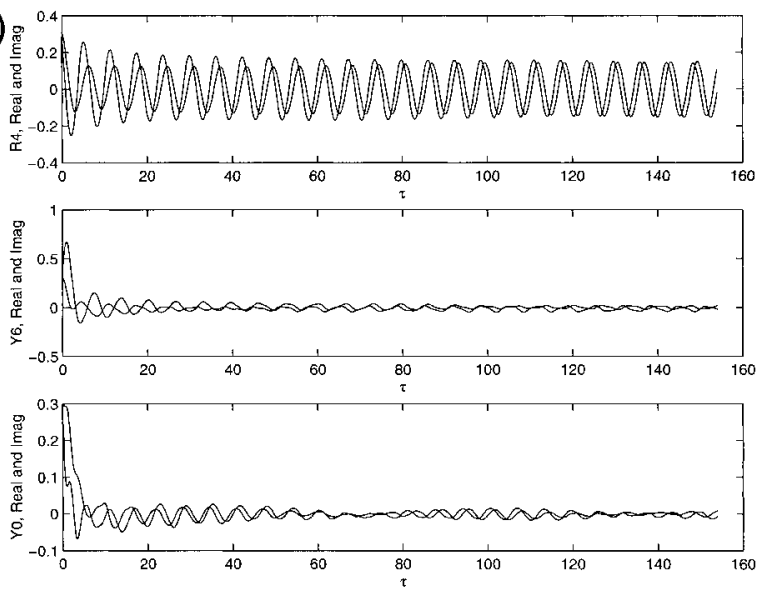

FIG. 9. An experiment on the first baroclinic mode. Even though the presence of multiple wave interactions makes this mode much richer than the barotropic, the actual results of a typical experiment are qualitatively similar, with convergence to a nonzero state, and a tendency of the modes with lowest wavenumbers to prevail. (a) Energy as a function of time with an initially fast decay and convergence to a nonzero final value. (b) Evolution of the various dispersive waves. The $k=4$ Rossby wave, which interacts with the longest $(k=1)$ mode of the Kelvin wave, converges to a much stronger final state than the other two modes. (c) Four snapshots of the Kelvin wave, which deforms as it moves at a nearly constant speed.
Equation (5.13) describes the evolution of the Kelvin wave over the slow time $\tau$. It is a Burgers equation forced by the nonlinear interaction between the Yanai wave and one mode of the topography. If the contribution from the Yanai wave is ignored, that is, $c_{3}=0$, the discussion in (5.10)-(5.13) provides a simplified asymptotic derivation of the nonlinear Kelvin wave.

\section{2) Evolution of the Yanai wave}

Here only a sketch will be presented of the salient features of the derivation of the equation in (1.2) for the Yanai wave satisfying the resonant conditions in (2.11) and (2.12). A complete detailed derivation is available upon request to the authors.

The critical coupling term involving topography and the Kelvin wave at order $\epsilon^{2}$ is given by the source term in the height equation for the linearized shallow water equation in (5.6), (5.7), and (5.8) with the form

$$
\left[h(x, y) e^{-y^{2} / 2} K(x-t, \tau)\right]_{x} .
$$

It is important to assess when this forcing term yields a resonant response from the linearized shallow water equations. With the expansion in parabolic cylinder functions, $D_{n}(y)$, given by

$$
h(x, y) e^{-\left(y^{2} / 2\right)}=\sum_{n=0}^{\infty} \tilde{h}_{n}(x) D_{n}(\sqrt{2} y)
$$

there is the corresponding series representation for (5.14),

$$
\begin{aligned}
& {\left[h(x, y) e^{-\left(y^{2} / 2\right)} K(x-t, \tau)\right]_{x}} \\
& \quad=\sum_{n=0}^{\infty}\left[\tilde{h}_{n}(x) K(x-t, \tau)\right]_{x} D_{n}(\sqrt{2} y) .
\end{aligned}
$$

The response of the Yanai waves from (5.6) to (5.8) to the forcing in (5.16) on the height equation is given by the equations (Gill 1982, chapter 11)

$$
\begin{aligned}
\left(\frac{\partial}{\partial t}+\frac{\partial}{\partial x}\right) q_{1}-\sqrt{2} v_{0} & =\left[\tilde{h}_{1}(x) K(x-t, \tau)\right]_{x} \quad \text { and } \\
\frac{\partial v_{0}}{\partial t}+\frac{1}{\sqrt{2}} q_{1} & =0
\end{aligned}
$$

where $q=u^{1}+\eta^{1}, v=v^{1}$, and the subscripts 0 and 


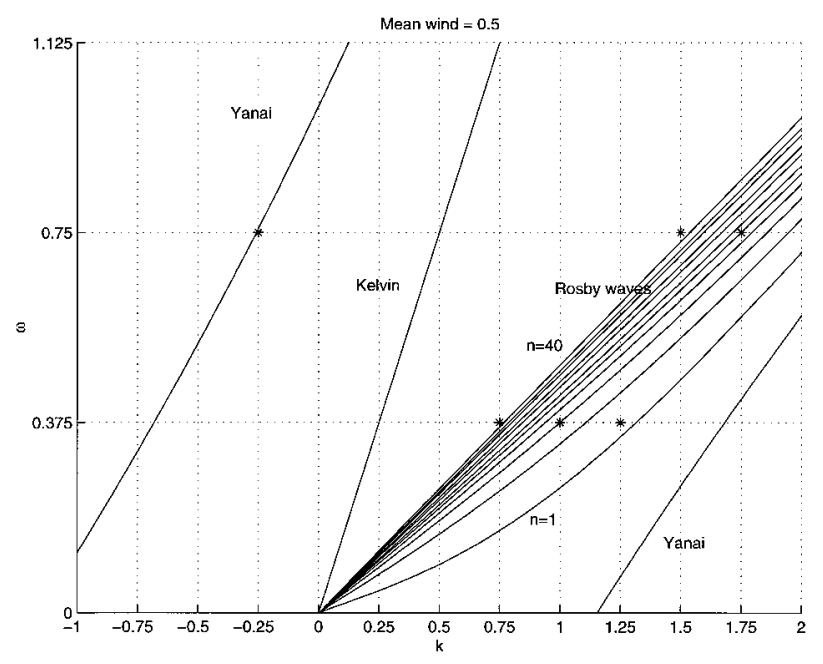

FIG. 10. Dispersion relation corresponding to an externally imposed uniform westerly mean wind of half the speed of a Kelvin wave. One Yanai mode and a cluster of Rossby waves resonate (or nearly resonate) with the Kelvin wave. This latter cluster of highly oscillatory Rossby waves suggests a midlatitude connection for the equatorial Kelvin wave.

1 stand for the corresponding terms in the parabolic cylinder expansion. In Fourier space, these equations yield the forced linear oscillator equations,

$$
\frac{\partial Y_{k}}{\partial t}+i \omega(k) Y_{k}=\sum_{j+l=k} i T_{k} \hat{\tilde{h}}_{1, j} \hat{K}_{l} e^{-i l t},
$$

with $\omega(k)$ satisfying the dispersion relation for Yanai waves,

$$
\omega(k)[\omega(k)-k]=1 .
$$

From (5.18), the response of the Yanai wave amplitude $Y_{k}$, at second order, to the interaction between topography and Kelvin waves will only be secular provided that

$$
j+l=k^{*} \quad \text { and } \quad l=\omega^{*},
$$

with $\omega^{*}$ satisfying (5.19). These are precisely the resonant conditions in (2.10) and (2.11) for this special case. One removes the secular terms in (5.18) by adding a slow time correction to the leading-order Yanai wave amplitude for the resonant mode $k$, as in the ansatz from (5.1) to (5.3), as demonstrated earlier for the Kelvin wave. Carrying out this procedure yields the following equation for the amplitude of the Yanai wave:

$$
d_{1} Y_{\tau}+d_{2} \hat{K}_{\omega^{*}}=0
$$

where

$$
\begin{aligned}
& d_{1}=\int_{-\infty}^{\infty} \frac{\omega^{*}+k^{*}}{\omega^{*}\left(k^{*}-\omega^{*}\right)}\left[1-\left(\omega^{*}-k^{*}\right)^{2}\right] e^{-y^{2}} d y \text { and } \\
& d_{2}=i \int_{-\infty}^{\infty} \frac{k^{*}\left(\omega^{*}-k^{*}\right)}{\omega^{*}} \hat{h}_{k^{*}-\omega^{*}}(y) y e^{-y^{2}} d y .
\end{aligned}
$$

This is an ordinary differential equation for the evo-

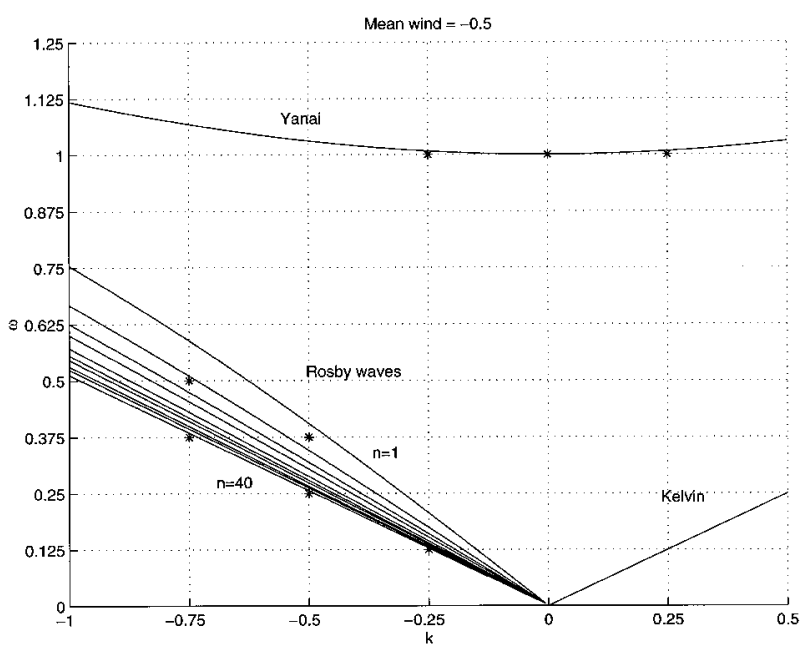

FIG. 11. Same as Fig. 10 but with an easterly wind. More Yanai modes resonate with the Kelvin wave, and again a cluster of Rossby waves do.

lution of the Yanai wave, as forced by a nonlinear interaction between the Kelvin wave and the topography.

Finally, it is convenient to rescale the variables in (5.13) and (5.21) to achieve the canonical form in (2.13) and (2.14). Notice that the independent variables $\theta$ and $\tau$ and the dependent variable $K$ can be rescaled only by positive real numbers in order not to alter the direction of time, while the variable $Y$ admits arbitrary complex rescalings. On the other hand, the coefficients $c_{1}$ and $c_{2}$ are real and positive, and even though $c_{3}$ and $d_{2}$ are complex, the product $\left(c_{3} d_{2}\right) /\left(c_{1} d_{1}\right)$ is real and positive. Hence, if rescaled variables are defined by

$$
\begin{aligned}
& K=\frac{c_{1}}{\alpha c_{2}}\left(\frac{c_{3} d_{2}}{c_{1} d_{1}}\right)^{1 / 2} \tilde{K}, \quad Y=\frac{c_{1} d_{2}}{\alpha c_{2} d_{1}} \tilde{Y}, \\
& \theta=\frac{1}{\alpha} \tilde{\theta}, \quad \text { and } \quad \tau=\left(\frac{c_{1} d_{1}}{c_{3} d_{2}}\right)^{1 / 2} \tilde{\tau},
\end{aligned}
$$

then by dropping the tildes, the canonical equations in (2.13) and (2.14) emerge.

\section{b. The first baroclinic mode}

The analysis of this case is entirely similar to the one developed above, with the added complexity that the various dispersive waves could interact among themselves, either directly or through the topography. However, for two waves to interact through the topography, their frequencies need to match, and this is not the case for the three dispersive waves included in section $2 \mathrm{~d}$. The derivation of the equations in (2.15) follows exactly the same path discussed above for the barotropic waves and the details are omitted. 


\section{THE NONLINEAR RESPONSE TO DIABATIC HEATING}

Throughout this paper, a wave speed, $c=50 \mathrm{~m} \mathrm{~s}^{-1}$, has been used to represent the first baroclinic mode in the shallow water model. This is the representative value utilized typically in studies concerning the response in the troposphere to diabatic heating from convection (Gill 1982; Yano et al. 1995; Milliff and Madden 1996) rather than the value of the dry Kelvin wave speed, $c$ $\simeq 150 \mathrm{~m} \mathrm{~s}^{-1}$ of the first baroclinic mode for the entire equatorial atmosphere (Kasahara and Puri 1981). To partially justify the use of this smaller wave speed, a theory for the nonlinear response to steady Gill forcing (Gill 1982 ) in the equatorial shallow water equations has been developed recently (Majda et al. 1999). This theory assumes that the heating is steady with small amplitude, $\epsilon \ll 1$, as in (5.5) above. The nonlinear response to this heating is a sum of two effects: the steady response, which decouples, and the transient response, which is advected by the steady response in the asymptotics. Thus, the nonlinear transient response satisfies the same simplified nonlinear equations with resonances as given in (3.1) and (3.2), and studied throughout this paper; the spectrum of the steady forcing generates the steady response spectrum, which plays an analogous role to the spectrum of the topography in (5.5) in determining the transient exchange of energy among various largescale equatorial waves through resonances. The details are too lengthy to present here and the interested reader can consult a recent extended abstract (Majda et al. 1999).

\section{Conclusions}

A new theoretical mechanism involving topographic resonance has been developed here where large-scale equatorial Kelvin waves can modify their speed through dispersion and interaction with other large-scale equatorial waves. These explicit resonance conditions have been presented in (2.11) and (2.12) and simplified reduced dynamical equations for the interaction have been derived in section 5 under the assumption of small amplitude waves and topography. The reduced dynamical equations corresponding to the barotropic and first baroclinic mode of the equatorial troposphere have been presented in section 2, and the detailed properties of solutions of these equations are studied in section 3. Explicit exact solutions in (3.6)-(3.8) as well as numerical experiments with the reduced dynamics indicate that this resonance mechanism can prevent the breaking of a propagating nonlinear Kelvin wave, slow down its speed, and concentrate most of its energy in large-scale zonal wavenumbers while simultaneously generating large-scale Yanai or Rossby modes with specific zonal wavelengths. The effect on topographic resonance of a strong eastward or westward mean flow with magnitude $25 \mathrm{~m} \mathrm{~s}^{-1}$ for waves corresponding to the first baroclinic mode is quite dramatic (see section 4) with the possi- bility of midlatitude connections to Rossby waves through this mechanism. In this paper, topography in the equatorial shallow water equations is utilized to crudely model large-scale land-sea contrasts, but the authors have no doubt that the role of topographic resonance, elucidated here, can be replaced in similar models by other large-scale inhomogeneous mechanisms involving convective parameterization (Neelin and Zeng 1997), which account for land-sea contrast in the Tropics. We plan to develop these more elaborate applications for one- and two-layer models in the near future.

Regarding various technical aspects, the work presented here, in order to focus on the basic mechanism, has intentionally ignored interesting related physical issues regarding near resonances as well as the equatorial three wave resonances (Ripa 1983a,b) and their competition with the topographic resonances presented here; this competition is especially interesting with the effect of a nonzero mean wind, as discussed in section 4 . The authors are currently pursuing these issues and will report on them elsewhere in the near future.

This work displays the potential importance of nonlinear effects interacting with large-scale dispersion for equatorial waves in the context of the equatorial shallow water equations. However, caution is necessary in interpreting these results for the fully stratified system since shallow water models yield an imperfect representation of both nonlinearity and radiation. Nevertheless, such simplified models might capture some of the essential features of the more complex dynamics.

Acknowledgments. The first author thanks Ralph Milliff for several stimulating conversations and for introducing him to the observational literature on the Madden-Julian oscillation. The research of Andrew J. Majda is partially supported by Grants NSF DMS-9596102001, NSF DMS-9625795, ONR N00014-96-0043, and ARO DAAG55-98-1-0129. The research of Rodolfo R. Rosales is partially supported by NSF Grant DMS9311438. The research of Cristina V. Turner is partially supported by a grant from the Antorchas Foundation. The research of Esteban G. Tabak is partially supported by an Alfred P. Sloan Research Fellowship and by NSF Grants DMS-9501073 and DMS-9701751.

\section{REFERENCES}

Andrews, P. G., J. Holton, and C. Leovy, 1987: Middle Atmosphere Dynamics. International Geophysics Series, Vol. 40, Academic Press, 489 pp.

Boyd, J. P., 1980: The nonlinear equatorial Kelvin wave. J. Phys. Oceanogr., 10, 1-11.

Celentano, C., 1995: Finite amplitude resonant acoustic waves without shocks. Ph.D. thesis, Massachusetts Institute of Technology, 198 pp.

Emanuel, K., 1987: An air-sea interaction model of intraseasonal oscillations in the Tropics. J. Atmos. Sci., 44, 2324-2340.

Gill, A. E., 1982: Atmosphere-Ocean Dynamics. Academic Press. Goswami, P., and B. N. Goswami, 1991: Modification of $n=0$ 
equatorial waves due to interaction between convection and dynamics. J. Atmos. Sci., 48, 2231-2244.

Kasahara, A., and K. Puri, 1981: Spectral representation of threedimensional global data by expansion in normal mode functions. Mon. Wea. Rev., 15, 1407-1436.

Madden, R. A., and P. Julian, 1972: Description of global-scale circulation cells in the Tropics with a 40-50-day period. J. Atmos. Sci., 29, 1109-1123.

—, and - 1994: Observations of the 40-50-day tropical oscillation-A review. Mon. Wea. Rev., 122, 814-836.

Majda, A., and R. Rosales, 1984: Resonantly interacting weakly nonlinear hyperbolic waves. Park I: A single space variable. Studies Appl. Math., 75, 117-148.

,-- , and M. Schonbek, 1988: A canonical system of integrodifferential equations arising in resonant nonlinear acoustics. Studies Appl. Math., 79, 205-262.

$\longrightarrow$, M. Shefter, and E. Tabak, 1999: The nonlinear response to Gill forcing in the Tropics. Extended Abstracts, 12th Conf. on At mosphere-Ocean Fluid Dynamics, New York, NY, Amer. Meteor. Soc., 161-162.

Milliff, R. F., and R. Madden, 1996: The existence and vertical structure of fast, eastward-moving disturbances in the equatorial troposphere. J. Atmos. Sci., 53, 586-597.

Neelin, D. J., and N. Zeng, 1997: The simplest quasi-equilibrium tropical circulation model-formulation. Extended Abstracts, Seventh Conf. on Climate Variations, Long Beach, CA, Amer. Meteor. Soc., 4-7.

_ , and J. Yu, 1994: Modes of tropical variability under convective adjustment and the Madden-Julian oscillation. Part I: Analytical theory. J. Atmos. Sci., 51, 1876-1894.

—, I. Held, and K. Cook, 1987: Evaporation-wind feedback and low-frequency variability in the tropical atmosphere. J. Atmos. Sci., 44, 2341-2348.

Ripa, P., 1982: Nonlinear wave-wave interactions in a one-layer reduced-gravity model on the equatorial $\beta$ plane. J. Phys. Oceanogr., 12, 97-111.

— 1983a: Weak interaction of equatorial waves in a one-layer model. Part I: General properties. J. Phys. Oceanogr., 13, 12081226.

, 1983b: Weak interaction of equatorial waves in a one-layer model. Part II: Applications. J. Phys. Oceanogr., 13, 1227-1240.

Shefter, M., 1997: Never breaking quasi-periodic solutions of weakly nonlinear gas dynamics. Ph.D. thesis, Massachusetts Institute of Technology, 109 pp.

Strang, G., 1968: On the construction and comparison of difference schemes. SIAM J. Num. Anal., 5, 506-517.

Takahashi, M., and B. A. Boville, 1992: Three-dimensional simulation of the equatorial quasi-biennial oscillation. J. Atmos. Sci., 49, 1020-1035.

Vaynblat, D., 1996: The strongly attracting character of large amplitude nonlinear resonant acoustic waves without shocks. A numerical study. Ph.D. thesis, Massachusetts Institute of Technology, $136 \mathrm{pp}$.

Wang, G., and Y. Xue, 1992: Behavior of a moist Kelvin wave packet with nonlinear heating. J. Atmos. Sci., 49, 549-559.

Yano, J., J. McWilliams, M. Moncrieff, and K. Emanuel, 1995: Hierarchical tropical cloud systems in an analog shallow-water model. J. Atmos. Sci., 52, 1723-1742. 